\title{
Enhanced processing speed in laser drilling of stainless steel by spatially and temporally superposed pulsed Nd:YAG laser radiation
}

\author{
K. Walther • M. Brajdic $\cdot$ E. W. Kreutz
}

Received: 25 May 2006 / Accepted: 28 August 2006/Published online: 8 November 2006

(C) Springer-Verlag London Limited 2006

\begin{abstract}
Percussion drilling through holes in stainless steel $(1.4301,5,8$, and $10 \mathrm{~mm}$ in thickness) was performed with the superposed radiation of two pulsed Nd:YAG lasers. Holes were drilled with flash lamp pumped $\mathrm{Nd}$ : YAG slab-laser radiation with a pulse duration of $0.5 \mathrm{~ms}$ superposed with diode-pumped solid-state (DPSS) laser radiation with a pulse duration of $17 \mathrm{~ns}$. The drilling efficiency is improved by the spatially and temporally superposed radiation of the two lasers. With the superposed laser radiation, drilling through stainless-steel samples at a maximum aspect ratio of 60 is performed up to four times faster with the reproducibility of the drilling time improved by a factor of six in standard deviation.
\end{abstract}

Keywords Laser drilling · Superposed laser beams . Drilling efficiency $\cdot$ Turbines $\cdot$ Cooling holes

\section{Introduction}

To enhance the efficiency of turbo-engines, the turbine temperature has increased drastically in the last decades due to new materials and cooling technologies. To further improve the efficiency of combined cycle power plants,

K. Walther $(\bowtie) \cdot$ M. Brajdic $\cdot$ E. W. Kreutz

Lehrstuhl für Lasertechnik,

Rheinisch-Westfälische Technische Hochschule Aachen,

Aachen, Germany

e-mail: Kurt.Walther@1lt.rwth-aachen.de

Present address:

E. W. Kreutz

Fraunhofer Institut für Lasertechnik,

Steinbachstraße 15,

52074 Aachen, Germany the aim is to increase the combustor outlet temperature up to $1,520^{\circ} \mathrm{C}$ in combination with a simultaneous reduction of the cooling fluid mass flow $[1,2]$. Both the improvements can be realized by an effusion cooling of the thermally highly loaded turbine components. The holes with a diameter of $0.2 \mathrm{~mm}$ are drilled by laser radiation at densities up to 100 holes per $\mathrm{cm}^{2}$. An inclination angle up to $60^{\circ}$ leads to a high aspect ratio, resulting in an extended processing time [3]. Due to the requirements for efficient laser drilling, much work is performed on understanding and improving the underlying processes [4-18].

The optimization of the drilling process regarding the productivity (e.g., drilling speed, maximum hole depth, operational capacity) or the quality (e.g., cylindricity, taper, recast) of the hole has been investigated with double-pulses $[7,8]$ and bursts of pulses [9-11]. A strong decrease in the number of pulses required to drill through stainless steel by coupling the laser radiation of a $0.15-\mathrm{ms}$ pulse with laser radiation of a pulse duration of $3.5 \mathrm{~ms}$ full-width at half maximum is reported by Lehane and Kwok [12]. The delay time between the two pulses has been varied showing as optimum delay to drill through by the irradiation of the laser pulse with small pulse duration subsequently after the laser pulse with large one. The enhancement of the drilling velocity is ascribed to the improved melt ejection due to the additional recoil pressure induced by the subsequent laser pulse [12].

The drilling speed and the reproducibility of the drilling time are investigated for stainless-steel samples with a thickness of 5, 8 , and $10 \mathrm{~mm}$ during the superposition of the laser radiation provided by a commercial flash lamp pumped Nd:YAG laser $(1,064 \mathrm{~nm}, 0.5 \mathrm{~ms})$ and a commercial diode-pumped solid-state (DPSS) laser $(1,064 \mathrm{~nm}, 17 \mathrm{~ns})$. The main topic of the investigations is directed towards the drilling speed, the geometry of the 
drills, and the reproducibility of the drill geometry in order to improve the productivity and the quality of laser drilling in applications.

\section{Experimental setup}

The experimental setup is shown schematically in Fig. 1, the specifications of the used lasers are given in Table 1. The spatial intensity profiles of the laser radiation are measured by photography using a camerabased monitoring system (MicrospotMonitor Co. PRIMES $\mathrm{GmbH})$. The radiation of both lasers exhibits a nearly circular Gaussian spatial intensity profile in the focus with comparable diameters. The linear polarised laser radiation of the LASAG Slab-Laser FM015, guided by two mirrors through a polarizer and a $\lambda / 4$-plate, is focused onto the sample by a lens with $100 \mathrm{~mm}$ focal length. The linear polarised laser radiation of the Lambda Physik DPSS-Laser Powergator 1064 is orthogonally aligned to the linear polarised laser radiation of the Slab-Laser. The laser radiation of both lasers is spatially coupled by the polariser. The Slab-Laser was operated at a pulse duration of $0.5 \mathrm{~ms}$, a pulse energy of $0.64 \mathrm{~J}$, and a repetition rate of $20 \mathrm{~Hz}$. The DPSS-laser is used at a repetition rate of $10 \mathrm{kHz}$ with the mean power varied by an external attenuator between 0 and $18 \mathrm{~W}\left(0 \leq \mathrm{P}_{\text {DPSS }} \leq 1.8 \mathrm{~mJ}\right)$ in steps of $\leq 5 \mathrm{~W}$. The actual mean power on the sample is measured by calorimetry. Both laser systems were not temporally synchronized.

The parameters used yield to a temporal overlap of 5 DPSS-laser ns-pulses during one 0.5-ms Slab-Laser pulse

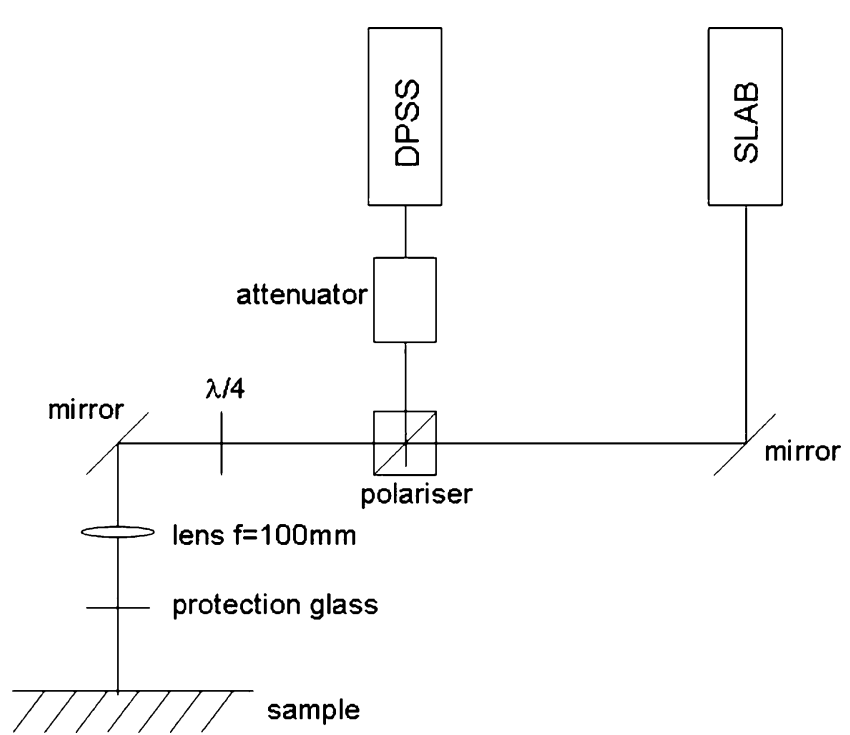

Fig. 1 Scheme of the experimental setup: the radiation of the DPSSlaser with a pulse duration of $17 \mathrm{~ns}$ is coupled by a polarizer with the radiation of the lamp-pumped Slab-Laser with a pulse duration of $0.5 \mathrm{~ms}$, without temporal synchronisation of the lasers
Table 1 Specifications of the lasers used

\begin{tabular}{lll}
\hline Laser & $\begin{array}{l}\text { Lamp-pumped } \\
\text { Slab FM015 }\end{array}$ & DPSS-Powergator \\
\hline Wavelength & $1,064 \mathrm{~nm}$ & $1,064 \mathrm{~nm}$ \\
Pulse duration & $0.5 \mathrm{~ms}$ & $17 \mathrm{~ns}$ \\
Pulse energy & $0.64 \mathrm{~J}$ & $0-1.8 \mathrm{~mJ}$ \\
Repetition rate & $20 \mathrm{~Hz}$ & $10 \mathrm{kHz}$ \\
Beam quality $\left(\mathrm{M}^{2}\right)$ & 2 & 1.7 \\
Focus diameter & $45 \mu \mathrm{m}$ & $42 \mu \mathrm{m}$ \\
Rayleigh length & $0.8 \mathrm{~mm}$ & $0.75 \mathrm{~mm}$ \\
\hline
\end{tabular}

(Fig. 2). The intensity of the DPSS-laser is up to two orders of magnitude larger than the Slab-Laser. The drilling was investigated with the focus of the Slab-Laser radiation at the top surface of the sample. The laser radiation of the two independent laser systems exhibits different beam parameters (waist diameter and divergence) and different distances of the beam waist to the focusing optic. This results in different waist diameters and divergences on the focusing lens. Using the same lens, the foci are shifted relative to each other depending on these parameters. The focus of the laser radiation of the DPSS-laser is located $4 \mathrm{~mm}$ below the Slab-Laser focus (Fig. 3). The holes are drilled in 5, 8, and 10 -mm-thick stainless-steel plates whereby the time to drill through has been recorded visually, with a precision of about $0.5 \mathrm{~s}$, as soon as a constant melt ejection is observed at the hole exit. For all experiments, oxygen at a pressure of 12 bar was used as the process gas.

\section{Results and discussion}

The percussion drilling experiments were carried out only with the laser radiation of the Slab-Laser to evaluate

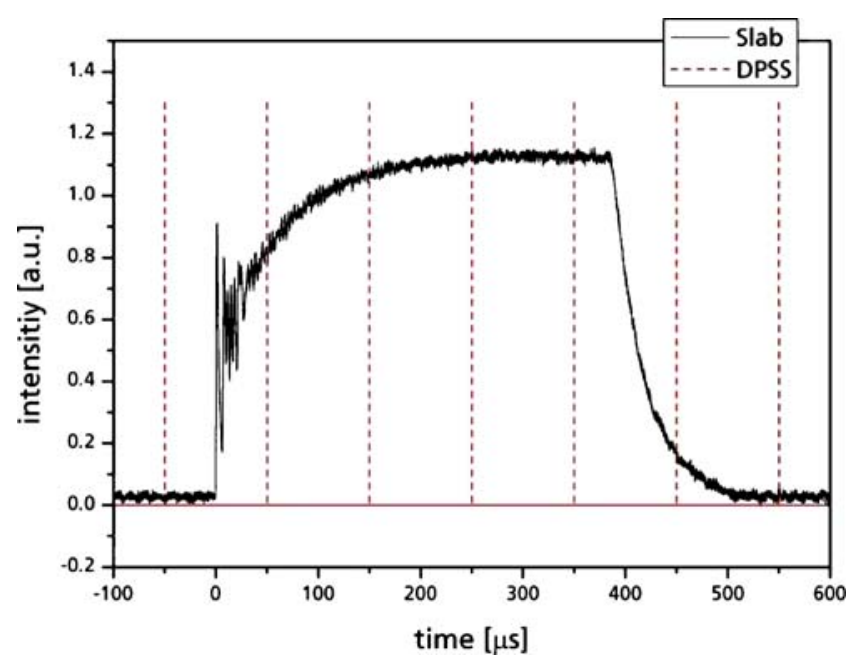

Fig. 2 Temporal intensity distributions of the Slab-Laser radiation and the DPSS-laser radiation 


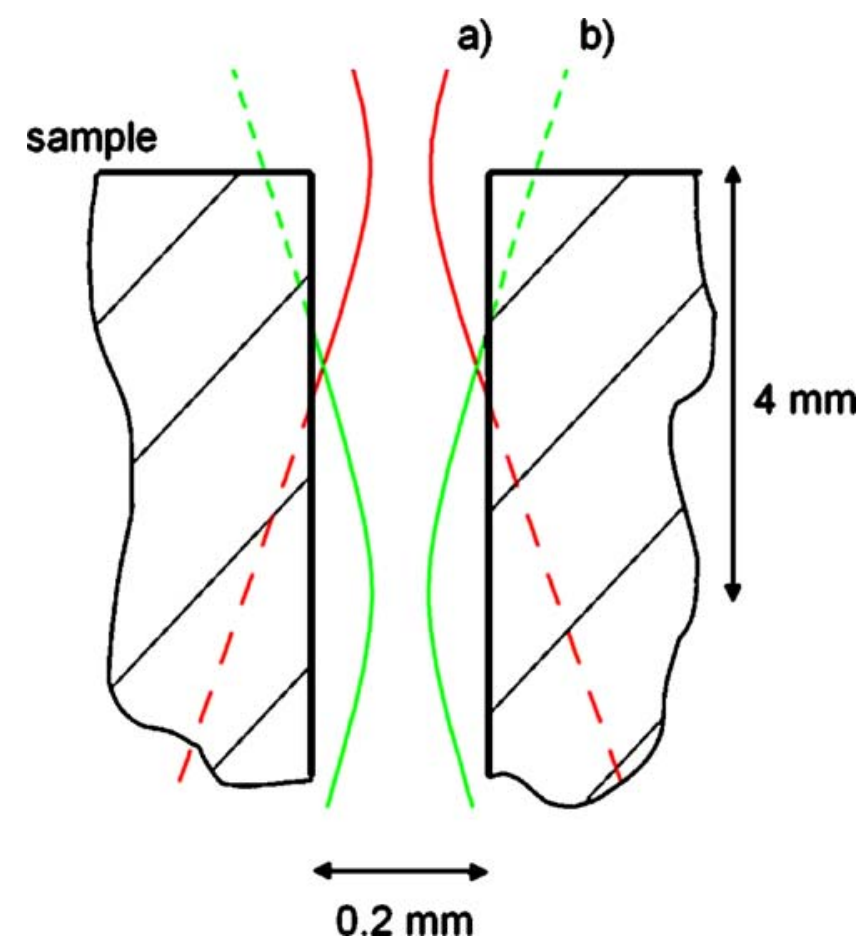

Fig. 3 Scheme of the entrance region of a drilled hole with the caustic of the superposed laser beams: a Slab-Laser; b DPSS-laser

reference times for drilling through the samples at different thicknesses (DPSS-laser mean power $\mathrm{P}_{\mathrm{DPSS}}=0 \mathrm{~W}$, Figs. 4 and 5). A sample with a thickness of $2 \mathrm{~mm}$ could not be drilled through using processing times up to $2 \mathrm{~min}$ by drilling only with the laser radiation of the DPSS-laser (Slab-Laser mean power $\mathrm{P}_{\text {Slab }}=0 \mathrm{~W}$ ). Due to this experimental result, no further investigations were carried out using only the laser radiation of the DPSS-laser.

The experiments with the laser radiation of the Slab-Laser superposed spatially and temporally by the laser radiation of the DPSS-laser were carried out using 5, 8, and 10-mm-thick

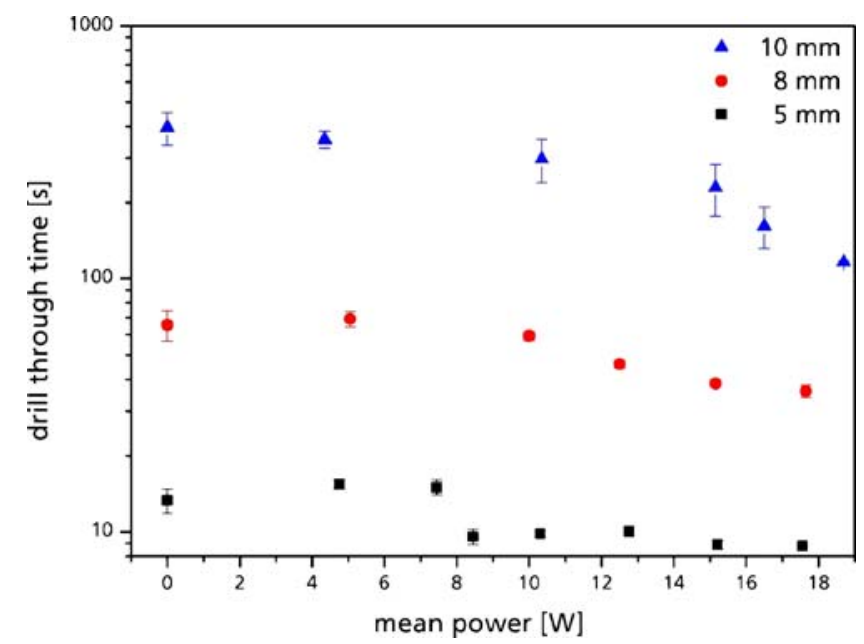

Fig. 4 Drill-through time of stainless-steel samples of various thickness as a function of the DPSS-laser mean power

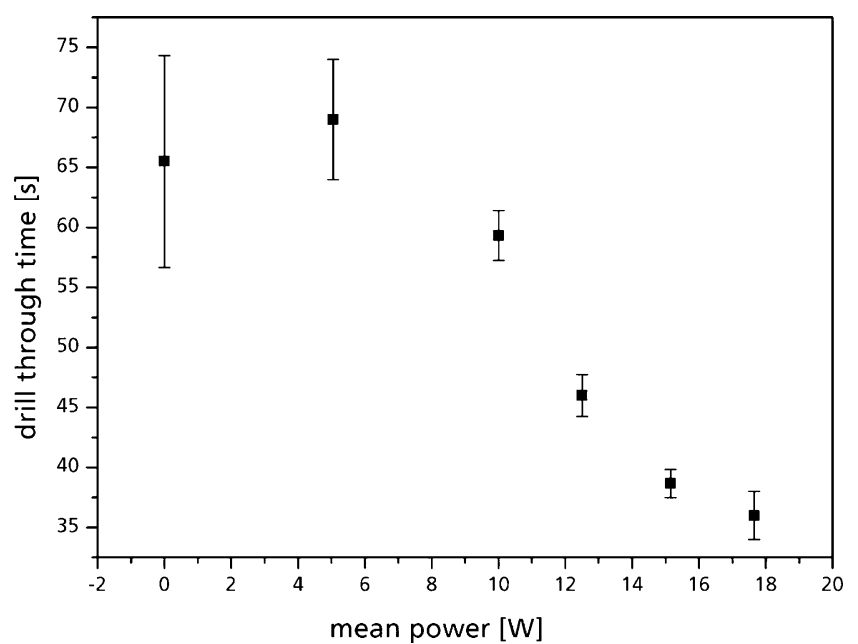

Fig. 5 Drill-through time of an $8-\mathrm{mm}$ steel sample as a function of the DPSS-laser mean power

stainless-steel plates from 0 to $18 \mathrm{~W}$ average power of the DPSS-laser (Fig. 4). Each data point represents the mean value of up to ten measurements and the corresponding standard deviation. The time to drill through decreases for all sample thicknesses investigated. The decrease compared to the reference time becomes larger with an increase in the sample thickness. Drilling through 8-mm-thick stainlesssteel plates results in a reduction of the mean drilling time from $66 \mathrm{~s}$ (by using only the radiation of the Slab-Laser) to $36 \mathrm{~s}$ with additional radiation of the DPSS-laser at $18 \mathrm{~W}$ mean power (Fig. 5). Simultaneously, the standard deviation of the mean drilling time is reduced from $25 \mathrm{~s}$ (drilling with only the Slab-Laser radiation) to $4 \mathrm{~s}$ (drilling with superposed DPSS-laser radiation at $18 \mathrm{~W}$ mean power).
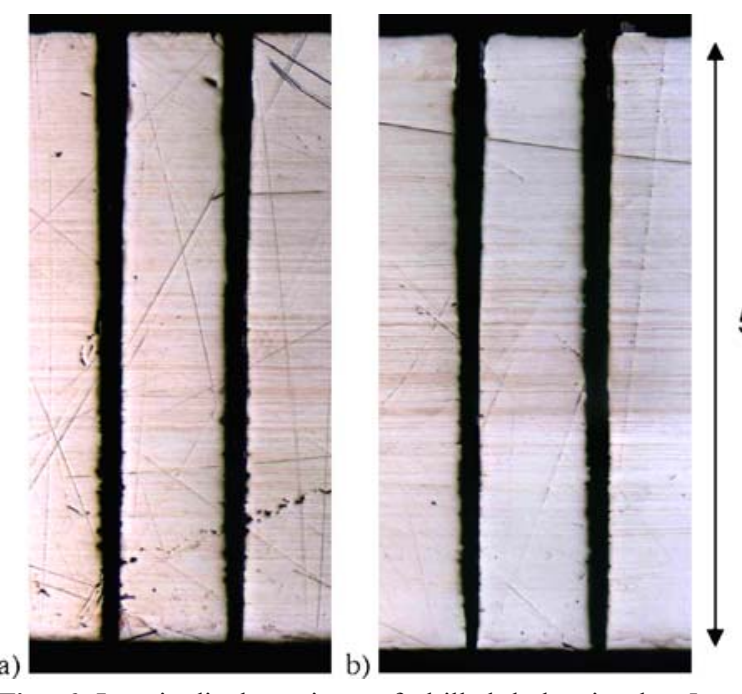

$5 \mathrm{~mm}$

Fig. 6 Longitudinal sections of drilled holes in the 5-mm steel samples without (a) and with the superposition (b) of the DPSS-laser radiation at a mean power of $18 \mathrm{~W}$. The hole diameters $(\mathbf{a}, \mathbf{b})$ are in the range of $0.17-0.18 \mathrm{~mm}$ with the geometric shape showing no dependence on the superposed radiation of the DPSS-laser 
Figure 6 shows the longitudinal sections of the holes in the $5-\mathrm{mm}$ plate. No influence on the geometric shape of the drilled hole due to the superposed laser radiation of the DPSS-laser can be detected; the diameters and the tapers are comparable.

The drilling process with the radiation of one laser pulse with a pulse duration in the $\mu$ s-regime can be described by a model representing four relevant process regimes [13]. The incident laser radiation is absorbed by the material resulting in melting, vaporization, and plasma formation at the ground of the developing hole (regime I). The recoil pressure induces an acceleration of the melt perpendicular to the incident laser beam. The heating of the molten material increases the diameter radially to the hole axis (regime II). The developing hole geometry and the recoil pressure lead to a coaxial melt flow towards the incident beam. With the melt flowing along the hole wall the melt cools down with a subsequent increase of viscosity and a decrease of velocity, leading to an increase of the thickness of the solidifying recast layer (regime III). With the further increase of thickness of the recast layer starting at a critical length, the incident beam can be shadowed, and a closure of the hole can occur (regime IV). Depending on the material properties and laser parameters during percussion, drilling closures develop at a critical hole depth. These closures can be removed again by subsequent laser pulses. With the further progression of the hole depth, the drilling process is maintained largely by multiple reflections of the laser radiation at the hole wall (Fig. 3). The spatial intensity profile is altered by reflection of laser radiation on the hole wall and the absorption decreases by the changing ground geometry [14]. Both processes lead inter alia to a modified melt dynamics causing the closures and a reduction of the drilling speed. These processes result in an unsteady or interrupted melt expulsion originating in different drill through times (DPSS-laser mean power $\mathrm{P}_{\mathrm{DPSS}}=0 \mathrm{~W}$, Figs. 4 and 5).

Explanations for the more efficient drilling with the spatially and temporally superposed radiation of the two lasers could be either the larger intensities caused by the DPSS-laser leading to a higher recoil pressure in the hole or an additionally heating of the vapour or the drill wall keeping the melt liquid and less viscous for enhanced times. Both processes result in a more effective melt expulsion. The larger recoil pressure causes a bigger acceleration of the melt in regime I. The additional heating leads to a delayed starting of the processes in regime III and to an extension of the critical length for the development of closures within the hole (regime IV). The temporal changes of the processes involved with the subsequent changes in matter properties yield in a modified formation and removal of closures within the hole.

\section{Conclusions}

Percussion drilling through holes in stainless steel $(1.4301,5$, $8,10 \mathrm{~mm}$ in thickness) was performed by superposed pulsed laser radiation provided by two Nd:YAG lasers with pulse durations of $0.5 \mathrm{~ms}$ and $17 \mathrm{~ns}$. The radiation of the lasers is spatially and temporally superposed. Due to the superposition of the radiation of the lasers, a remarkable decrease in the drilling time and in the deviation of the mean drilling time can be achieved. The formation of closures within the hole is modified; the melt ejection is more continuous and the drilling is more reproducible. The longitudinal sections show no influence on the geometric shape of the drilled hole due to the superposed laser radiation of the DPSS-laser. The diameters and the tapers are comparable to the conventional percussion drilled holes by the Slab-Laser.

More detailed experiments are necessary to assign the efficiency improvement to the processes during laser percussion drilling described. Further metallographic and microscopic analysis will give more information on the processes involved. An analysis with coaxial photography using a high-speed camera will allow a more precise interpretation of the processes during the drilling, especially the influence of the superposed laser radiation on the formation and removal of closures within the hole.

Acknowledgements The authors gratefully acknowledge the financial support by the German Research Foundation (DFG) within the Collaborative Research Centre (SFB) 561 "Thermally highly loaded, porous and cooled multilayer system for combined cycle power plants" and the basic research project HSD "High speed drilling with pulsed laser radiation: modeling and diagnosis of processes". The authors are very thankful to Prof. W. Schulz for the stimulating discussions regarding the presentation "Drilling model for microsecond-pulses" at CLEO 2005 in Munich. The authors are very indebted to Dr. A. Horn for helpful discussions with respect to the experimental results and their interpretation.

\section{References}

1. Framework of the Collaborative Research Centre (SFB) 561 (1998) Thermally highly loaded, porous and cooled multilayer system for combined power plants. RWTH Aachen, Aachen

2. Boyce MP (2001) Gas turbine engineering handbook, second edn. Incompressible flow turbomachines. Gulf Professional Publishing, Houston

3. Lugscheider E, Bobzin K, Maes M, Lackner K, Poprawe R, Kreutz EW, Willach J (2005) Laser drilled microholes in zirconiacoated surfaces using tow variants to implement the effusion cooling of first-stage turbine blades. Adv Eng Mater 7/3:145-152

4. Ng GKL, Li L (2002) Optimisation of laser percussion drilling process for improved repeatability. Proc ICALEO (CD-ROM), Scottsdale

5. Migliore L, Kardos G, Ozkan A, Derkach O, Schaeffer R, Dunsky C (2004) Laser choices for micromachining: drilling speed and quality comparison. Proc ICALEO (CD-ROM), San Francisco 
6. Breitling D, Ruf A, Dausinger F (2004) Fundamental aspects in machining of metals with short and ultrashort laser pulses. Proc SPIE 5339:49-63

7. Forsman A, Banks P, Perry M, Campbell E, Dodell A, Armas M (2005) Double-pulse machining as a technique for the enhancement of material removal rates in laser machining of metals. J Appl Phys 98:033302

8. Hartmann C, Gillner A, Aydin Ü, Noll R, Fehr T, Gehlen C, Poprawe R (2005) Increase of laser micro ablation rate of metals using multi-pulses. Proc COLA (in press)

9. Ostermeyer M, Kappe P, Menzel R, Sommer S, Dausinger F (2005) Laser drilling in thin materials with bursts of Ns-pulses generated by stimulated brillouin scattering (SBS). Appl Phys A 81:923-927

10. Lapczyna M, Chen KP, Herman PR, Tan HW, Marjoribanks RS (1999) Ultra high repetition rate (133 MHz) laser ablation of aluminum with 1.2-ps pulses. Appl Phys A 69:883-886

11. Stoian R, Boyle M, Thoss A, Rosenfeld A, Korn G, Hertel I (2002) Laser ablation of dielectrics with temporally shaped femtosecond pulses. Appl Phys Lett 80:353-355
12. Lehane C, Kwok H (2001) Enhanced drilling using a dual-pulse Nd:YAG laser. Appl Phys A 73:45-48

13. Schulz W (2005) Diagnosis and modelling of nonlinear dynamics in laser cutting, welding and drilling. Mater Res Soc Symp Proc 850:MM5.2.1-MM5.2.13

14. Ruf A, Berger P, Dausinger F, Hügel H (2004) Analytical investigations on geometric influences on laser drilling. J Phys D Appl Phys 34: 2918-2925

15. Low D, Li L, Byrd P (2004) The influence of temporal pulse train modulation during laser percussion drilling. Opt Lasers Eng 35:149-164

16. Low D, Li L (2002) Comparison of intra- and interpulse modulation in laser percussion drilling. Proc Inst Mech Eng 216:167-171

17. Low D, Li L (2002) Effects of inter-pulse and intra-pulse shaping during laser percussion drilling. Proc SPIE 4426:191-194

18. Klimentov S, Garnov S, Kononenko T, Konov V, Pivovarov P, Dausinger F (1999) High-rate deep-channel ablative formation by picosecond-nanosecond combined laser pulses. Appl Phys A 69:633-636 